\title{
Study of Defect Coalescence in Heterogeneous Material Systems using Broad Band Dielectric Spectroscopy
}

\author{
VAMSEE VADLAMUDI*, MUTHU RAM PRABHU ELENCHEZHIAN, \\ PRIYANSHU KUMAR BANERJEE, RASSEL RAIHAN, and \\ KENNETH REIFSNIDER
}

Proceedings of the American Society for Composites-Thirty-second Technical Conference

DOI $10.12783 /$ asc2017/15260

\begin{abstract}
Composite materials are essential for many modern applications, including airplanes and cars, energy conversion and storage devices, medical prosthetics, and civil structures. Detecting the initiation, growth, accumulation, and coalescence of micro-damage in these heterogeneous materials and predicting the onset of component failure using conformal Broadband Dielectric Spectroscopy (BbDS) is a promising area of ongoing research. Recently, the authors have developed the critical path concept and Heterogeneous fracture mechanics concept that depicts the effect of defect nucleation, growth, coalescence, and fracture plane development and correlation of these damage mechanisms to change in dielectric response respectively. Current research applies those concepts to detect the damage mode and the critical fracture path (conduction path) that leads to eventual failure. Also, the use of BBDS to detect the weak adhesion bonding is proposed, where the change in dielectric properties as a function of the frequency of the applied potential through the thickness of the sample is measured in the vicinity of the weak region. It is observed that at low frequency the gradient in the potential indicates a spike which marks the charge concentration around the imperfect region.
\end{abstract}

University of Texas Arlington, Mechanical and Aerospace Engineering, $701 \mathrm{~S}$ Nedderman Dr, Arlington, TX 76019, U.S.A. 


\section{INTRODUCTION}

Unlike metallic materials, engineered materials (e.g. composites) are designed to develop distributed damage consisting of various types of defects and even multiple breaks in the same reinforcing fiber. Local changes in the material state also have significant effects on the prognosis of the future behavior of a composite material system. Therefore, the primary interest is not in single local events but in the evolution process of multiple events that have a collective global effect. The first question is how to measure something that is sensitive to the damage details in the final phase of the life, and how to interpret such measurements in such a way that we can use those interpretive models to extract a "warning" of impending failure.

Under combined applied field conditions, materials degrade progressively. To evaluate such material state changes there are many tools and methods but most of them do not give a direct and quantitative assessment of the damage state. Broadband Dielectric Spectroscopy (BbDS) is a robust tool to extract the material-level information, including the morphology changes caused by micro-detect generation and the orientation of those defects. Fazzino et al. [1] and Raihan et al. [2] have shown that the association of response to a dielectric field with the response to a mechanical field for a material element is direct.

To illustrate this, Reifsnider et al. [3] manually introduced sequential damage in the form of random matrix cracking followed by fiber fracture as shown in Figure 1 (left) and calculated the resulting change in electric compliance (capacitance) shown in Figure 1 (right). This was simulated by introducing moist air in the crack openings and fiber fracture regions.

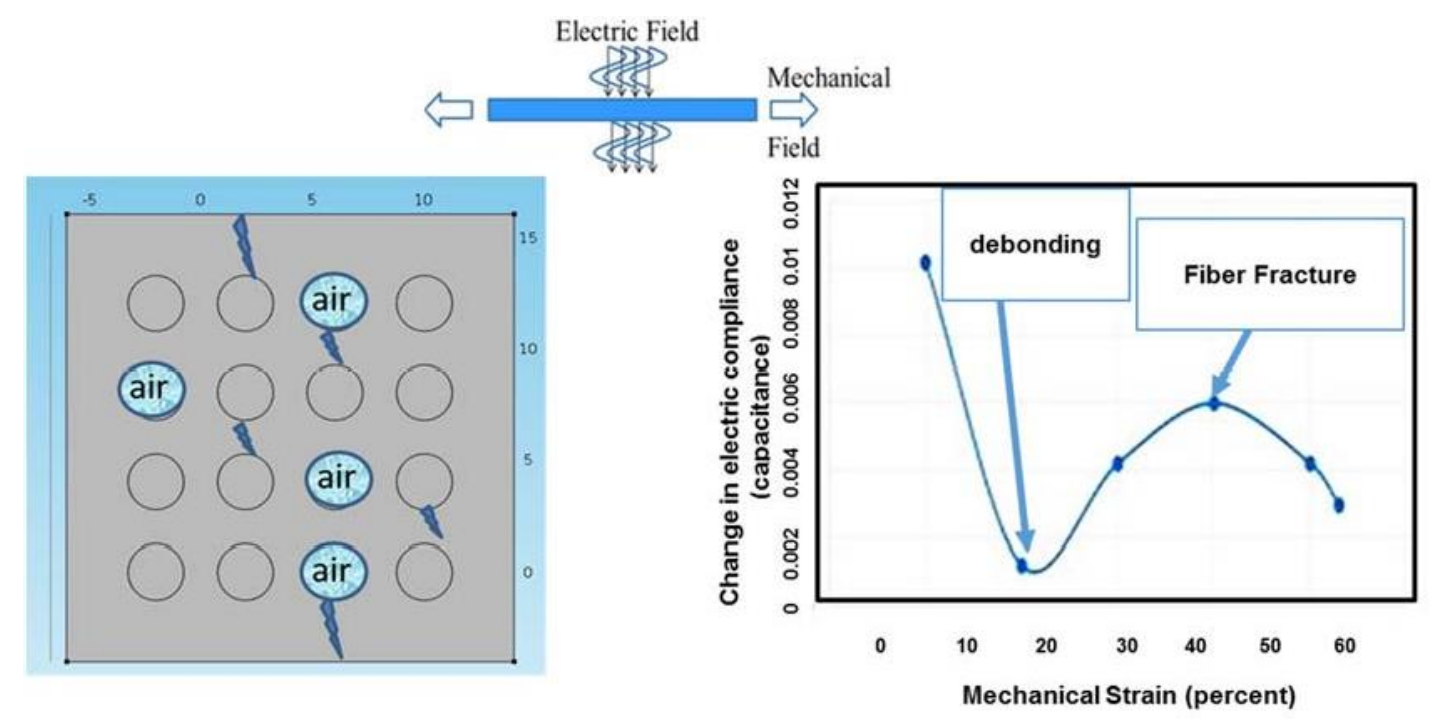

Figure 1 Model illustrating matrix cracks and fiber fracture (left) and change in electrical compliance (capacitance) (right) 
The foundation for the concept of heterogeneous fracture mechanics was based on these physical evidences and dielectric measurements [1-3]. Further, from data obtained by Raihan et al. [2] for woven glass fiber composite coupons loaded at 45 degrees to the principal fiber axis, as shown in figure 2, the change in stored electrical energy $\left(U_{E}\right)$ and change in released mechanical energy $\left(U_{M}\right)$ with respect to the change in defect area $(\partial \mathrm{A})$ were calculated using the relations shown in Eq. (1) and Eq. (2) respectively.

$$
\begin{aligned}
& 6 \underset{6 \mathrm{U}}{\underline{\mathrm{E}}}=\frac{\mathrm{E}^{2}}{2} \frac{6 \mathrm{~s}}{\partial Æ} \\
& \frac{6 \mathrm{U}_{\mathrm{M}}}{6 \rightleftarrows}=\frac{\mathrm{P}^{2} \frac{6 \mathrm{c}}{2}}{\bar{\partial} \mathrm{E}} \mathrm{G}
\end{aligned}
$$

where $\mathrm{E}$ is the applied vector electric field (through thickness in this case), $\mathrm{s}$ is the effective permittivity of the material, $A$ is the area between the parallel plates, $G$ is the strain energy release rate, $\mathrm{P}$ is the mechanical load, and $\mathrm{c}$ is the material mechanical compliance for linear response. In a dielectric study we generally measure total capacitance, $\mathrm{C}$, between two parallel plates separated by the specimen thickness (d) by

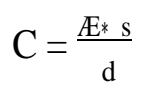

The measurement reflects the effective permittivity (s) of the material between two parallel plates of area, A, over the thickness, d. Combining eq. $(1,3)$ we calculate the change in stored electrical energy.

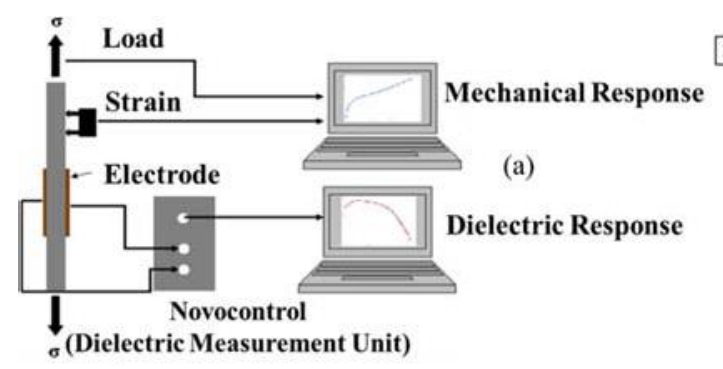

(a)

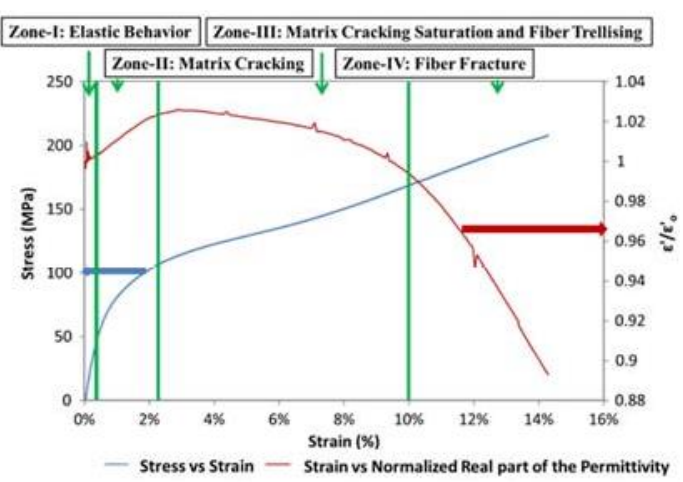

(b)

Figure 2, (a) In-Situ tensile test setup, (b) Mechanical and Dielectric response of the coupon 


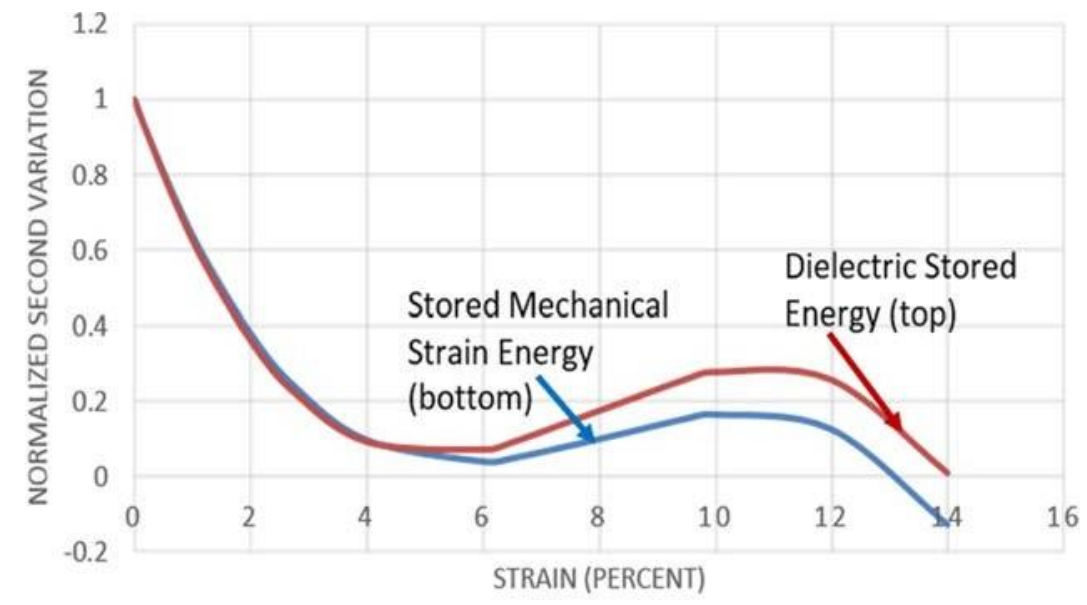

Figure 3, Comparison of the second variation (variation from linear slope) of the stored strain energy and dielectric capacitance.

From the experimental data, we plot the second variation of strain energy and the second variation of the measured capacitance with strain; normalizing both of those plots by the initial value, it was observed that the two variations were remarkably similar as shown in figure 3 . We believe that the physics of damage initiation (microcrack formation) events drives corresponding changes in strain energy and dielectric response measured in the laboratory, i.e., that those observables are dual responses to the process of damage development.

The current paper focuses on application of this concept to various material systems and on understanding how the physics can be effectively modeled to simulate the experimental behavior.

\section{OPEN HOLE TENSION MODEL}

Heterogeneous fracture mechanics was applied to simulate an open hole tension testing of a coupon made of neat epoxy resin. Mechanical loading was applied using Abaqus $^{\mathrm{TM}}$ and those results were used in a subsequent dielectric study which was performed using COMSOL MULTIPHYSICS ${ }^{\mathrm{TM}}$. The frame work is shown below in figure 4. Uniaxial displacement was applied in the X direction and a 1Volt AC signal was applied perpendicular to the loading direction through the thickness, as shown in figure 5. The homogeneous material system meant that there would be no damage progression implying once the damage initiates it would immediately fail [4]. The hole in the coupon acted as the stress concentrator. The tensile strength of the resin was taken to be $60 \mathrm{MPa}$ and the $\mathrm{G}_{\text {Ic }}$ value was taken to be $0.177 \mathrm{~N} / \mathrm{mm}$ [5]. The crack path and the force displacement curve are shown in figure $6(\mathrm{a} \& \mathrm{~b})$ respectively. 


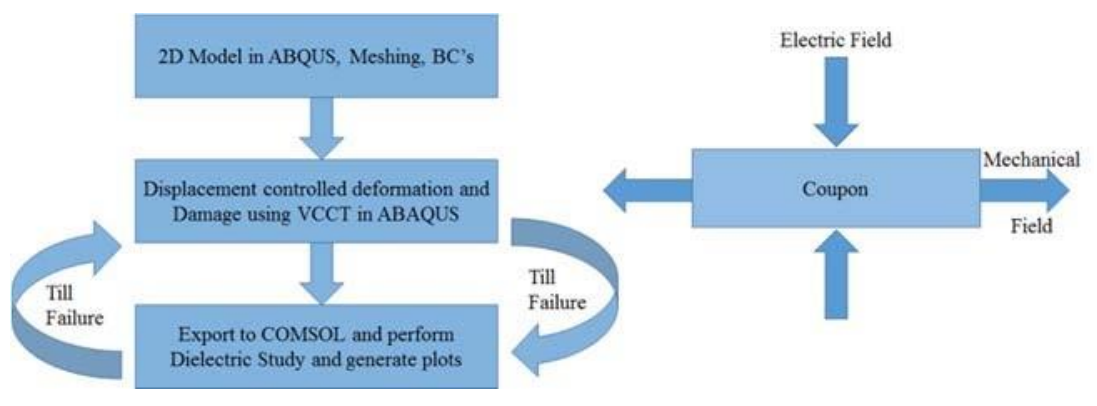

Figure 4, Current framework of the simulation

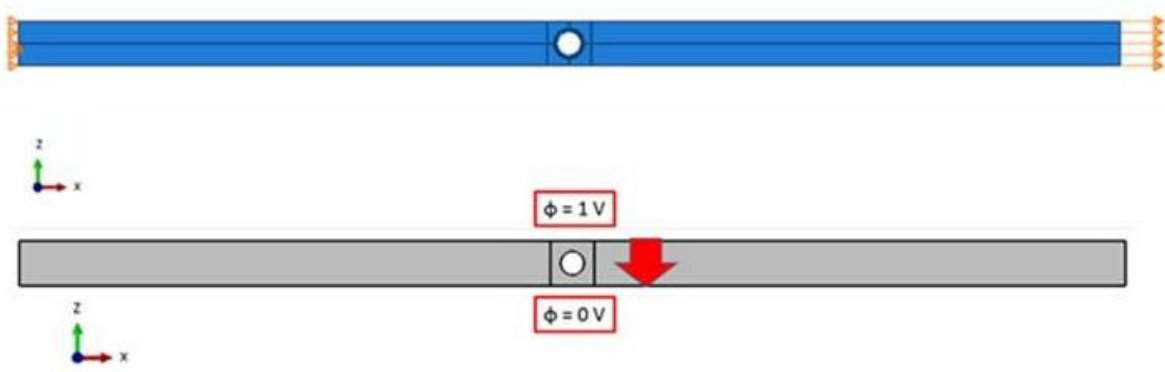

Figure 5, Mechanical and electrical boundary conditions for the open hole tension coupon modeled in this analysis.

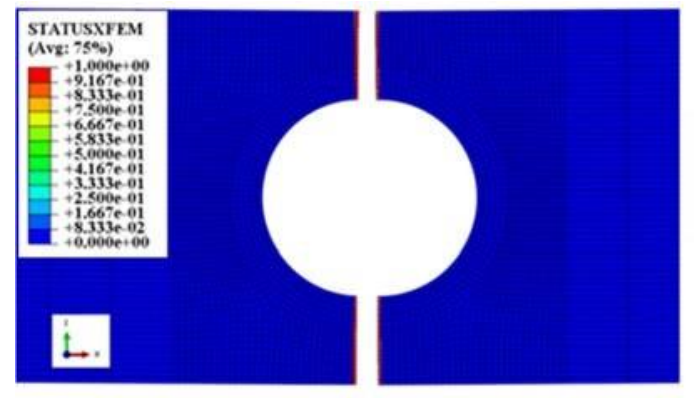

(a)

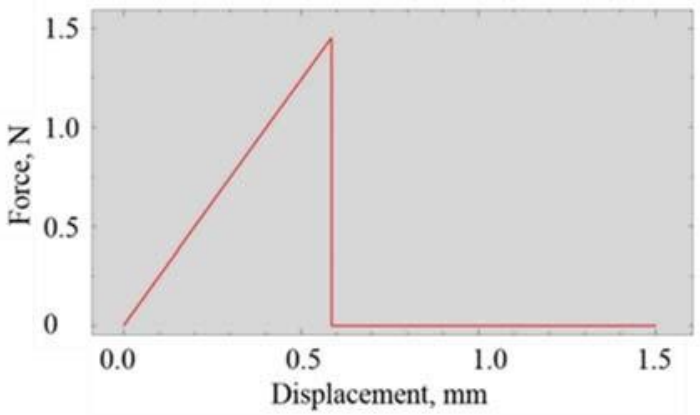

(b)

Figure 6, (a) Fracture path obtained from the simulation, (b) Force-Displacement response of the coupon 


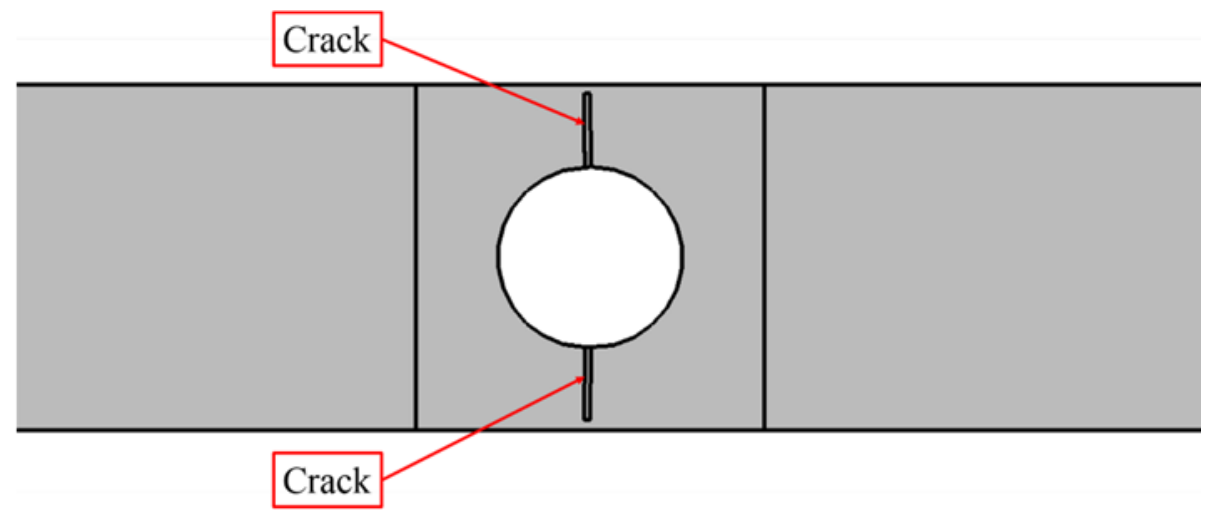

Figure 7, Deformed model imported in to COMSOL MULTIPHYSICS ${ }^{\mathrm{TM}}$ (zoomed in to the vicinity of the crack).

The deformed geometry at several load increments are then imported to COMSOL MULTIPHYSICS ${ }^{\mathrm{TM}}$ for the conformal dielectric study. The imported model with cracked geometry is shown in figure 7 (zoomed in to the vicinity of the crack).

Property degradation helps us to model the mechanical damage by varying the properties in the damaged regions. When we import the deformed specimen into COMSOL MULTIPHYSICS ${ }^{\mathrm{TM}}$ the only way to indicate damage is by varying material properties. In all material systems, depending on the working environment, moisture can either be absorbed or released [6] and hence we assume that moisture occurs inside the crack domains during damage. The dielectric properties of neat resin and moisture are tabulated in Table I.

TABLE I, DIELECTRIC PROPERTIES OF NEAT EPOXY \& MOISTURE

\begin{tabular}{|ccc|}
\hline Property & Epoxy & Moisture \\
\hline Conductivity & $1.64 \mathrm{E}-17 \mathrm{~S} / \mathrm{mm}$ & $5 \mathrm{E}-7 \mathrm{~S} / \mathrm{mm}$ \\
Permittivity & 3.15 & 20 \\
\hline
\end{tabular}

The dielectric impedance is calculated and plotted at various frequencies and at various a/w_ratios (ratio of crack length to width) of the coupon as shown in figure 8(a). It was observed that with increasing damage the calculated impedance decreased owing to the creation of a conduction path, and at fracture the response was driven by conduction owing to the creation of the least resistance path.

In order to better interpret the dielectric data, it is generally recommended to normalize it such that we capture the relative change in state of the material. An example of the calculation of the normalized parameter used in the present work is shown below.

Consider, the impedance at the initial undeformed state as $\mathrm{Z}_{0}$, the normalized impedance will be calculated as

$$
\operatorname{norm} \mathrm{Z}=\frac{\mathrm{Z}}{\mathrm{Z}_{0}}
$$


For the initial undeformed state norm $\mathrm{Z}$ will be 1 ; the variation in normalized impedance at various excitation frequencies for different $\mathrm{a} / \mathrm{w}$ ratios is shown in figure 8 (b). It was observed that only at low frequencies in the range $1 \mathrm{mHz}$ to $10 \mathrm{~Hz}$ the change in material state was captured, and at higher frequencies the response is similar at all frequencies. It was also observed that this phenomenon was driven by the relaxation time of the local charge accumulation, i.e., the time required for the charges to redistribute. Hence, at higher frequencies there is not sufficient time for the local charge accumulation to redistribute around the newly created surfaces and hence the method is unable to capture any change in the material state.

However, in the current analysis, because of the homogeneous material system, the requirement was only to find the precursor for the damage initiation as there would be no damage progression. It was observed that the dielectric response had a change in slope just before damage initiation which was not recorded in the force displacement response as shown in figure 9.

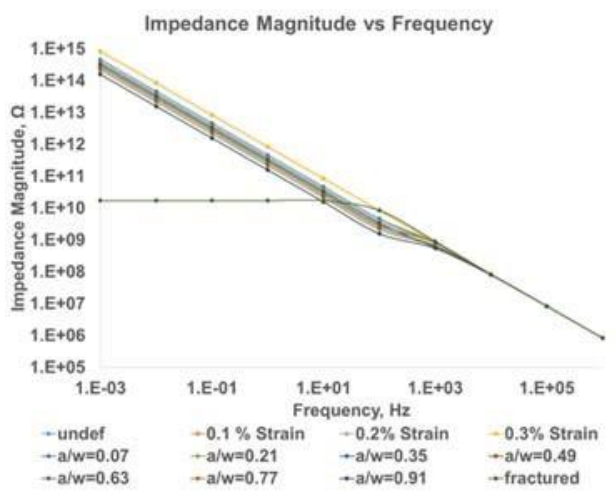

(a)

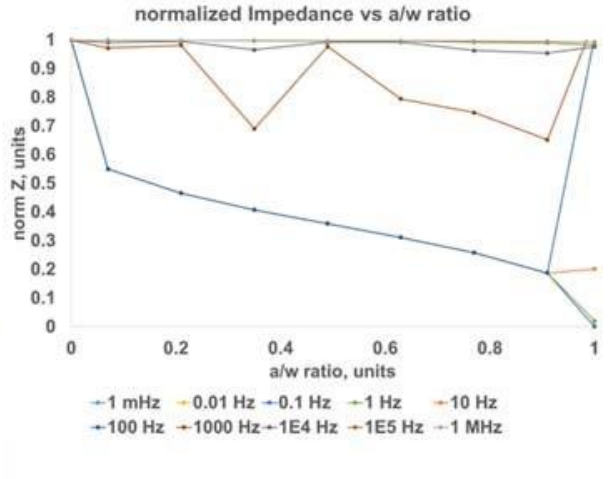

(b)

Figure 8, (a) Variation of impedance with frequency at various a/w ratios, (b) Variation of normalized impedance with $\mathrm{a} / \mathrm{w}$ ratio at different frequencies. 


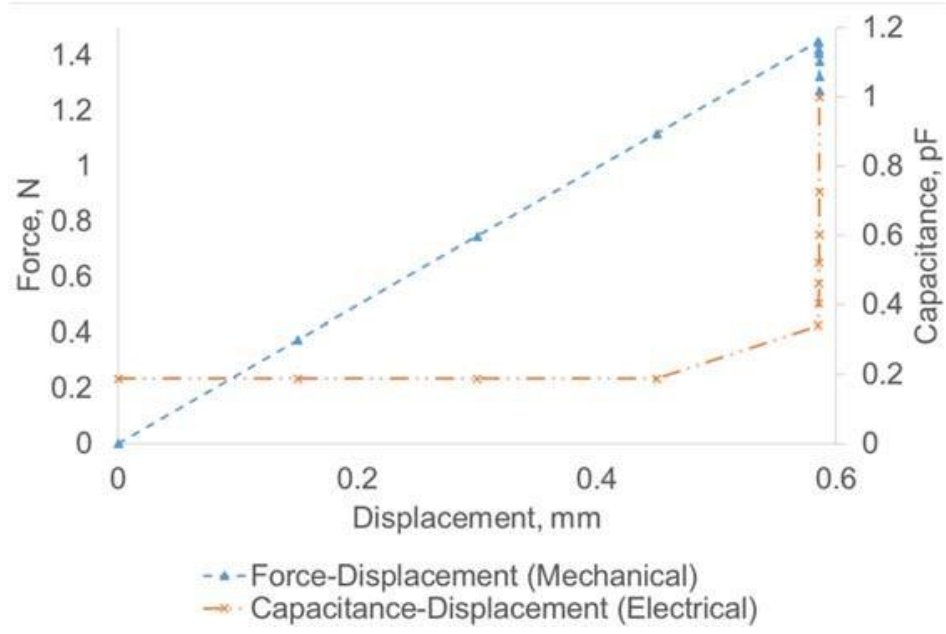

Figure 9, Force-Displacement response and Capacitance-Displacement response of the material system just before failure.

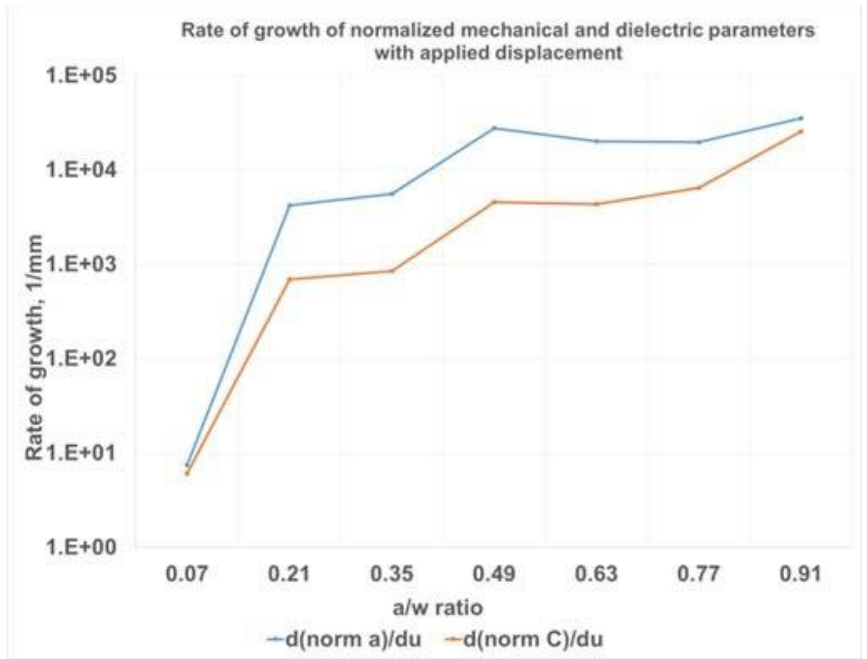

Figure 10, normalized variation of crack growth and dielectric capacitance with displacement 
Hence, it was observed that clearly the dielectric response was able to determine the initiation of damage in the model. To better correlate the relation between these two responses we plotted the normalized variation of crack growth and capacitance with displacement. It was observed that the variation in both the responses were quite similar, as shown in figure 10 . Hence, our results show that the damage induced by mechanical loading was captured by the charge redistribution at low frequencies in the dielectric response.

\section{CONCLUSIONS AND FUTURE WORK}

From our earlier experimental work we had derived the concept of heterogeneous fracture mechanics and then applied it to an isotropic homogeneous material system. In the present work we have introduced crack growth calculated in a homogeneous material with nonuniform stress state through the thickness using Abaqus ${ }^{\mathrm{TM}}$, and then imported those sequential changes into COMSOL to calculate the corresponding changes in the dielectric properties of the material in that region in response to an applied low-frequency electric field. It was observed that the damage induced by the mechanical loading correlated with the induced charge redistribution around the newly formed crack surfaces, and that the technique was able to capture the change in material state with the formation of a conduction path created by the growth of the crack through the thickness. Also, with this technique we were able to identify a damage precursor in the changes of the dielectric response that marked the onset of fracture, which was the breakthrough from the analysis approach.

However, we would also like to validate the concept with heterogeneous material systems and by using BbDS to find damage precursors that would help us detect the change in material state before the structure loses its required structural integrity.

\section{REFERENCES}

[1]. Fazzino, P., Reifsnider, K., Majumdar, P. (2009). "Impedance spectroscopy for progressive damage analysis in woven composites," Composites Science and Technology, 69: 2008-2014.

[2]. Raihan, R., Adkins, J.M., Baker, J., Rabbi, F., Reifsnider, K. (2014). "Relationship of dielectric property change to composite material state degradation," Composites Science and Technology, 105: 160-165.

[3]. Reifsnider, K., Raihan, R., Vadlamudi, V. (2016). "Heterogeneous fracture mechanics for multidefect analysis," Composite Structures, 156: 20-28.

[4]. Vadlamudi, V., Raihan, R. and Reifsnider, K. (2017). "Multiphysics Based Simulation of Damage Progression in Composites," Materials Sciences and Applications, 8: 389-404.

[5]. Iarve, E.V., Gurvich, M.R., Mollenhauer, D.H., Rose, C.A., Dávila, C.G. (2011). "Meshindependent matrix cracking and delamination modeling in laminated composites," International journal for numerical methods in engineering, 88: 749-773.

[6]. Shen, C.H., Springer, G. S. (1976). "Moisture absorption and desorption of composite materials," Journal of Composite Materials, 10: 2-20.

Vadlamudi, Vamsee, et al. 2017. "Study of Defect Coalescence in Heterogeneous Material Systems using Broad Band Dielectric Spectroscopy," Proceedings of the American Society for Composites: Thirty-Second Technical Conference, Purdue University, West Lafayette, Indiana, October 23-25, 2017. Lancaster, PA, USA: DEStech Publications, Inc., Electronic product-9 pp. 